\title{
Efectos de metales pesados, glifosato y sus mezclas binarias sobre el crecimiento de algas verdes
}

\author{
Constanza Afione Di Cristofano; Ángela B. Juárez²3; Juan Moretton ${ }^{12}$ \& \\ Anahí Magdaleno, ${ }^{1, \mathbb{U}}$ \\ ${ }^{1}$ Universidad de Buenos Aires, Facultad de Farmacia y Bioquímica, Cátedra de Salud Pública e Higiene Ambiental. \\ ${ }^{2}$ Universidad de Buenos Aires, Facultad de Ciencias Exactas y Naturales, Departamento de Biodiversidad y Biología \\ Experimental. ${ }^{3}$ CONICET-Universidad de Buenos Aires, Instituto de Biodiversidad y Biología Experimental y Aplicada
} (IBBEA).

Resumen. Los ríos y arroyos de zonas rurales de la Provincia de Buenos Aires contienen concentraciones variables de metales pesados y glifosato. En el presente estudio se evaluó la toxicidad de los metales $\mathrm{Cu}$, $\mathrm{Pb}$ y $\mathrm{Zn}$, el herbicida glifosato (producto activo y formulación ATANOR ${ }^{\circledR}$ ) y sus mezclas binarias, en dos especies de algas verdes (una estándar [Raphidocelis subcapitata] y una cepa autóctona de Scenedesmus acutus aislada del Arroyo Burgos, Buenos Aires). Los bioensayos se realizaron en un rango de concentración entre $0.5 \mathrm{mg} / \mathrm{L}$ y $20 \mathrm{mg} / \mathrm{L}$, y a los 7 días de incubación se estimó la densidad algal. Para obtener las concentraciones efectivas que inhiben el 10, 20 y 50\% del crecimiento (CE10, CE20 y CE50) de cada sustancia individual y de las mezclas mediante un ajuste no lineal se modeló el porcentaje de inhibición del crecimiento (\%I) en función de cada concentración. Según las CE50 obtenidas, la toxicidad del Cu y del Zn fue mayor en $R$. subcapitata $(7.47 \pm 2.14$ y $6.51 \pm 2.26 \mathrm{mg} / \mathrm{L}$, respectivamente) que en $S$. acutus $(10.90 \pm 3.75$ y $>20 \mathrm{mg} / \mathrm{L})$. El Pb y el glifosato no resultaron tóxicos para ninguna de las dos cepas. El glifosato ATANOR ${ }^{\circledR}$ fue tóxico solamente para $R$. subcapitata $(C E 50=12.00 \pm 3.10 \mathrm{mg} / \mathrm{L})$. Según los valores de CE10 y CE20 de las sustancias individuales y de las mezclas binarias, la cepa $S$. acutus resultó más sensible. Teniendo en cuenta las unidades tóxicas (UT) obtenidas, se evidenció que las mezclas $\mathrm{Cu}+\mathrm{Zn}, \mathrm{Cu}+$ glifosato ATANOR® y $\mathrm{Zn}+$ glifosato ATANOR® presentaron efectos antagónicos sobre $R$. subcapitata (según valores de UT obtenidos a partir de las CE20 y CE50), mientras que las mezclas $\mathrm{Cu}+\mathrm{Pb}$ y $\mathrm{Cu}+\mathrm{Zn}$ tuvieron efectos sinérgicos y antagónicos, respectivamente, sobre $\mathrm{S}$. acutus (según valores de UT obtenidos a partir de las CE20). Este estudio destaca la importancia de realizar bioensayos con sustancias tóxicas utilizando cepas algales autóctonas que permiten inferir efectos potenciales en las comunidades y tramas tróficas nativas.

[Palabras clave: cobre, plomo, zinc, toxicidad]

Aвstract. Effects of heavy metals, glyphosate and their binary combinations on the growth of green algae. Rivers and streams of rural areas of the Buenos Aires Province contain variable concentrations of heavy metals and glyphosate. In this study, the toxicity of the metals $\mathrm{Cu}, \mathrm{Pb}$ and $\mathrm{Zn}$, the herbicide glyphosate (active product and ATANOR ${ }^{\circledR}$ formulation) and their binary combinations, was assessed on two green algae species (the standard species Raphidocelis subcapitata and a native strain of Scenedesmus acutus isolated from Burgos Stream [Buenos Aires]). The bioassays were carried out applying a concentration range of $0.5 \mathrm{mg} / \mathrm{L}-20 \mathrm{mg} / \mathrm{L}$, in incubations lasting 7 days after which the algal density was estimated. To obtain the effective concentrations inhibiting 10, 20 and $50 \%$ of the growth (EC10, EC20 and EC50) of individual substances and their combinations, the percentage of growth inhibition (\%I) was modeled as a function of each concentration by a nonlinear fit. According to the EC50 obtained for each species, the toxicity of $\mathrm{Cu}$ and $\mathrm{Zn}$ was higher in R. subcapitata $(7.47 \pm 2.14$ and $6.51 \pm 2.26 \mathrm{mg} / \mathrm{L}$, respectively) than in S. acutus $(10.90 \pm 3.75$ and $>20 \mathrm{mg} / \mathrm{L})$, while $\mathrm{Pb}$ and glyphosate active compound were not toxic for neither of the two strains. ATANOR® glyphosate was toxic only to $R$. subcapitata $(E C 50=12.00 \pm 3.10 \mathrm{mg} / \mathrm{L})$. According to the EC10 and EC20 values of the individual substances and the binary combinations, S. acutus showed higher sensitivity than R. subcapitata. From the analysis of toxic units (TU), the combinations $\mathrm{Cu}+\mathrm{Zn}, \mathrm{Cu}+\mathrm{glyphosate} \mathrm{ATANOR}^{\circledast}$ and $\mathrm{Zn}+\mathrm{glyphosate} \mathrm{ATANOR}^{\circledast}$ showed antagonistic effects on $R$. subcapitata (according to the TU values obtained from EC20 and EC50), while the $\mathrm{Cu}+\mathrm{Pb}$ and $\mathrm{Cu}+\mathrm{Zn}$ combinations showed synergistic and antagonistic effects, respectively, on S. acutus (according to the TU values obtained from the EC20). These results highlight the importance of conducting bioassays of toxic substances using native algal strains which allow inferring potential effects in native communities and food webs.

[Keywords: copper, lead, zinc, toxicity]

Editora asociada: María Diéguez 


\section{INTRODUCCIÓN}

Las actividades industriales y agropecuarias generan sustancias contaminantes que se distribuyen en distintos ambientes y afectan la salud de los ecosistemas y del hombre (Jorgensen et al. 1997). En la Argentina, la agricultura representa una importante fuente de producción primaria y secundaria, para la cual se destinan 31 millones de hectáreas. Los cultivos transgénicos (soja, maíz y algodón) abarcan las tres cuartas partes de la tierra cultivada donde se utilizan herbicidas, especialmente el glifosato, para controlar las malezas durante el cultivo y los períodos de barbecho (Sarandón 2013). Según Aparicio et al. (2013), el glifosato adsorbido a las partículas del suelo puede trasladarse mediante procesos de escorrentía hacia los cursos de agua donde puede acumularse en los sedimentos y luego desorberse y pasar a la fase líquida.

El glifosato (N-fosfonometilglicina) es un herbicida no selectivo post-emergente cuyo principal mecanismo de acción consiste en inhibir la enzima 5-enolpiruvil-shiquimato-3fosfato sintetasa, responsable de la formación de los aminoácidos aromáticos fenilalanina, tirosina y triptofano en las plantas (Duke 1988). El impacto del glifosato en sus diferentes formulaciones sobre organismos 'no blanco' ha sido objeto de amplia investigación a lo largo de la última década. Se reportaron evidencias de efectos fisiológicos en algas verdes, tales como estrés oxidativo, reducción de la actividad superoxidodimutasa y catalasa, además de inhibición del crecimiento (Romero et al. 2011). Por otra parte, existe evidencia que el glifosato y sus formulaciones Roundup ${ }^{\circledR}$ y Atanor $^{\circledR}$ afectan la estructura y función del fitoplancton y del perifiton y, por lo tanto, la calidad del agua y los ecosistemas de agua dulce (Pérez et al. 2007; Vera et al. 2010; Pizarro et al. 2015; González et al. 2019). Cabe destacar que en aguas superficiales de ríos y arroyos de la provincia de Buenos Aires se determinaron concentraciones de glifosato entre 13 y 700 mg/ L (Peruzzo et al. 2008; Bollani et al. 2019).

Asimismo, en zonas agrícolas se detectaron concentraciones de metales pesados probablemente provenientes de agroquímicos, fertilizantes fosfatados y abonos orgánicos (Fergusson 1990; Alloway 1995; Pignata et al. 2002), de las aguas residuales utilizadas para riego y de las deposiciones atmosféricas (Pignata et al. 2002; Kim et al. 2015). Las concentraciones elevadas de metales pesados en el medio acuático pueden producir efectos adversos en las algas, tales como la reducción de la biosíntesis de clorofila, inhibición de la fotosíntesis y del crecimiento (Omar 2002; Küpper et al. 2002).

Los bioensayos de laboratorio permiten analizar la biodisponibilidad y los efectos tóxicos interactivos de las sustancias sobre los organismos acuáticos (Nowell et al. 2014). Las algas fotosintéticas en particular son modelos seleccionados en ensayos ecotoxicológicos debido a que tienen crecimiento rápido y pueden cultivarse en laboratorio utilizando medios sintéticos que permiten mantenerlas en condiciones fisiológicas óptimas. En cuanto a su importancia ecológica, las algas son los principales productores primarios del fitoplancton y la base de las cadenas tróficas acuáticas. La especie estándar propuesta como organismo de referencia por varias agencias ambientales es el alga verde Pseudokircheriella subcapitata (USEPA 2002; Environmental Canada 2007; ISO 2009). Actualmente, la identidad taxonómica válida para esta especie es Raphidocelis subcapitata, según la investigación genómica realizada por Suzuki et al. (2018). Esta especie es sensible a numerosos agentes tóxicos, como metales pesados y pesticidas (Blinova 2004; Guéguen et al. 2004; Kahru et al. 2005; Magdaleno et al. 2015). Sin embargo, los efectos de los contaminantes sobre una especie estándar no necesariamente son los mismos que los que ocurren en cepas de especies aisladas de cuerpos de agua de una zona de interés. Las cepas autóctonas pueden proporcionar información relevante que permita inferir los efectos de los contaminantes en su medio ambiente (Magdaleno et al. 2014; Carusso et al. 2018).

Por otra parte, en los ambientes naturales, los organismos vivos se encuentran expuestos simultáneamente a una mezcla compleja de compuestos que pueden interactuar de manera aditiva, antagónica o sinérgica. De este modo, la inclusión del análisis de los efectos de las mezclas de dos o más contaminantes sobre los organismos vivos resulta necesaria para evaluar con más precisión los riesgos de exposición en el ambiente natural. Estudios recientes detectaron la presencia de concentraciones elevadas de metales pesados cobre $(\mathrm{Cu})$, plomo $(\mathrm{Pb})$ y cinc $(\mathrm{Zn})$ y de glifosato en aguas del Arroyo Burgos, un cuerpo de agua localizado en una zona agrícola-ganadera de la provincia de Buenos Aires (Bollani et al. 2019; Magdaleno et al. 2018). En este marco, esta investigación 
tiene el objetivo de evaluar los efectos de los metales $\mathrm{Cu}, \mathrm{Pb}$ y $\mathrm{Zn}$, y del glifosato (principio activo y formulación ATANOR $^{\circledR}$ ) y de sus mezclas binarias sobre el crecimiento de dos especies de algas verdes: una cepa estándar (Raphidocelis subcapitata) y una cepa autóctona de la especie Scenedesmus acutus aislada del arroyo Burgos.

\section{Materiales y Métodos}

Las cepas de las dos especies de algas verdes, $R$. supcapitata (Korshikov) y S. acutus (Meyen) (Chlorococcales, Chlorophyta) fueron obtenidas de la Colección de Cultivos del Laboratorio de Ficología, Departamento de Biodiversidad y Biología Experimental de la Facultad de Ciencias Exactas y Naturales, Universidad de Buenos Aires. La cepa de la especie estándar proviene originalmente de la Colección de Cultivos de Algas y Protozoos (CCAP) con la identificación 278/4. La cepa autóctona de la especie $S$. acutus fue aislada del arroyo Burgos (San Pedro, Provincia de Buenos Aires) y cultivada axénicamente con la identificación BAFC CA 14.

Se prepararon soluciones madre de $1 \mathrm{~g} / \mathrm{L}$ de $\mathrm{Cu}^{2+} / \mathrm{CuSO}_{4^{\prime}} \mathrm{Pb}^{2+} \mathrm{Pb}\left(\mathrm{NO}_{3}\right)_{2}, \mathrm{Zn}^{2+} / \mathrm{ZnSO}_{4}$, glifosato (sal de isopropilamina de Nfosfonometil glycina) y de la formulación ATANOR $^{\circledR}$, que contiene $48 \%(\mathrm{p} / \mathrm{v})$ de glifosato. A partir de estas soluciones se realizaron diferentes diluciones en Medio Basal de Bold (BBM) para el crecimiento de las algas (Archibald and Bold 1970), a fin de obtener las siguientes concentraciones de ensayo en mg/L: 0.5, 1, 2.5, 5, 7.5, 10 y 20. Las concentraciones de metales pesados se midieron por espectrofotometría de absorción atómica y las concentraciones de glifosato (principio activo) y glifosato ATANOR ${ }^{\circledR}$ se midieron por medio de cromatografía líquida de alta eficacia (HPLC). Según estas determinaciones, hubo una similitud cercana al $95 \%$ entre las concentraciones nominales y las concentraciones reales de manera que en todo el trabajo se consideraron las primeras.

Para realizar los bioensayos se usaron placas de 96 pozos, según el protocolo estandarizado por Environmental Canada (2007), utilizando un volumen final de $200 \mu \mathrm{L}$ en cada pozo (20 $\mu \mathrm{L}$ de inóculo+180 $\mu \mathrm{L}$ de tóxico o mezcla). Una modificación del protocolo fue la inclusión del medio BBM en lugar del propuesto por la agencia canadiense, debido a que el BBM contiene los mismos nutrientes, pero en mayor concentración, condición más parecida a los cuerpos de agua eutrofizados de la Provincia de Buenos Aires. El inóculo inicial fue de $1 \times 10^{5}$ células/mL. Cada ensayo fue realizado por cuadruplicado. El control se realizó con un total de 8 repeticiones con medio BBM, sin añadir tóxico. Las mezclas binarias se realizaron incorporando partes iguales de cada sustancia (1:1) a fin de obtener las mismas concentraciones finales que en el caso de las sustancias individuales. Las placas se colocaron en un agitador orbital termostatizado $\left(22^{\circ} \mathrm{C}\right)$, a $80 \mathrm{rpm}$ de velocidad y con luz continua durante 7 días. La duración del ensayo se estableció en 7 días ya que en ese período se observó un crecimiento poblacional máximo (hasta $1 \times 10^{7}$ células/mL) de las algas en los controles en las condiciones mencionadas. Al finalizar el ensayo se estimó la densidad algal mediante lecturas de absorbancia a una longitud de onda de $620 \mathrm{~nm}$, utilizando un espectrofotómetro de lectura de placas. Esta estimación se realizó en base a una curva de calibración estándar para cada especie de alga ajustando los datos de absorbancia y concentración celular con un modelo lineal de primer orden $\left(\mathrm{R}^{2}>0.95\right.$ [Carusso et al. 2018]). El ensayo se repitió entre 5 y 7 veces para cada tratamiento y cada cepa algal.

A partir de los datos de absorbancia se armó una matriz y se aplicó estadística descriptiva, ANOVA de un factor y contrastes a posteriori mediante la prueba de Tukey para evaluar diferencias significativas entre las absorbancias de cada tratamiento y el control. Se utilizó el programa estadístico R. Para cada concentración de tóxico se obtuvieron los porcentajes de inhibición (\%I) con respecto al control, según:

$$
\% \mathrm{I}=100 \times[\mathrm{C}-\mathrm{T}] / \mathrm{C}
$$

donde \%I es el porcentaje de inhibición del crecimiento algal, C es la absorbancia media de los controles y T la absorbancia media en cada tratamiento. Se probaron distintos modelos a fin de seleccionar el mejor ajuste para explicar los datos observados. Se seleccionó un modelo no lineal y se confeccionaron gráficos mediante el programa GraphPadPrism. La función no lineal utilizada se define como la siguiente ecuación sigmoidea:

$$
\% \mathrm{I}=100 \times\left(\mathrm{a}^{\mathrm{HS}} / 1+\mathrm{X}^{\mathrm{HS}}\right)
$$

donde $X$ es la concentración del tóxico o mezcla en $\mathrm{mg} / \mathrm{L}$, a corresponde al valor medio entre $0 \%$ y $100 \%$ de inhibición del crecimiento 
de las algas, y HS es la pendiente que describe la inclinación de la curva. El ajuste se realizó utilizando el método de cuadrados mínimos. A partir de los parámetros de ajuste se estimaron las concentraciones efectivas de cada compuesto o mezcla que producen una inhibición del crecimiento del 10, 20 y 50 por ciento (CE10, CE20 y CE50, respectivamente).

Para evaluar si el efecto combinado de las dos sustancias de cada mezcla fue aditivo, sinérgico o antagónico se utilizó el concepto de unidad tóxica (UT), según Broderius et al. (2005), el cual asume que cada sustancia componente de la mezcla tiene el mismo modo de acción tóxica. El efecto conjunto se describe de la siguiente manera: si UT $=1 \pm 0.2$, la toxicidad de la mezcla es aditiva (adición de concentración), si UT $<0.8$ representa sinergia potencial (más que aditivo), y si UT $>1.2$ indica antagonismo potencial (menos que aditivo) (González-Pleiter et al. 2013). Para el cálculo de las UT de las mezclas se utilizó la siguiente ecuación:

$\mathrm{UTmezcla}=\mathrm{UTa}+\mathrm{UTb}$

donde, UTa es la unidad tóxica del componente a de la mezcla y UTb, la unidad tóxica del componente $\mathrm{b}$. Las UT de cada componente $(\mathrm{a}$ y $\mathrm{b})$ se calcularon según la siguiente ecuación:

$$
\mathrm{UT}=\mathrm{Ci} / \mathrm{CE} 50
$$

donde, Ci es la concentración del componente i (a o b) que produce una inhibición del 50\% cuando ambos componentes están presentes en la mezcla (las CE50 de la mezcla), y CE50, la concentración inhibitoria 50 del componente $\mathrm{i}(\mathrm{a}$ o $b)$.

\section{Resultados}

De los tres metales analizados, el $\mathrm{Cu}$ y el $\mathrm{Zn}$ resultaron tóxicos para $R$. subcapitata, el $\mathrm{Cu}$ resultó tóxico para $S$. acutus, mientras que el $\mathrm{Pb}$ no afectó a ninguna de las dos especies (Tabla 1). En cuanto al glifosato, la formulación ATANOR ${ }^{\circledR}$ resultó tóxica para la especie estándar, mientras que el principio activo no resultó tóxico para las especies estudiadas. Teniendo en cuenta las CE50 para el $\mathrm{Cu}$ y el Zn, R. subcapitata presentó valores más bajos que $S$. acutus, lo que indicaría que la especie estándar es más sensible a estos metales. Sin embargo, según surge de comparar las CE10 y CE20, la sensibilidad de la especie autóctona fue mayor a bajas concentraciones de las sustancias ensayadas (Tabla 1). En ambas especies se observó un efecto dosisrespuesta para el $\mathrm{Cu}$ y el $\mathrm{Zn}$, mientras que en $R$. subcapitata los aumentos progresivos en las concentraciones de $\mathrm{Pb}$ no inhibieron su crecimiento (Figura 1). No se obtuvo un buen ajuste a la función no lineal en $S$. acutus para el $\mathrm{Pb}\left(R^{2}=0.3\right)$; los \% I fueron inferiores al $50 \%$ con una pendiente asociada cercana a 0 (HS=$0.38 \pm 0.15)$, lo que indica una relación no lineal muy baja entre los \%I y las concentraciones de $\mathrm{Pb}$ (Tabla 2). El glifosato ATANOR ${ }^{\circledR}$ inhibió significativamente a $R$. subcapitata $(P<0.05)$, mientras que la exposición al principio activo

Tabla 1. Concentraciones efectivas 10, 20 y 50 (CE10, CE20 y CE50) de cada sustancia individual obtenidas para la cepa de la especie Raphidocelis subcapitata y la cepa de la especie Scenedesmus acutus. Las concentraciones de las mezclas (1: 1) se expresan en $\mathrm{mg} / \mathrm{L}$.

Table 1. Effective concentrations 10, 20 and 50 (EC10, EC20 and EC50) of each individual substance obtained for the strain of Raphidocelis subcapitata and the strain of Scenedesmus acutus. The concentrations of the mixtures (1:1) are expressed in $\mathrm{mg} / \mathrm{L}$.

\begin{tabular}{lccc}
\hline Tóxico & CE10 & CE20 & CE50 \\
\hline R. subcapitata & & & \\
$\mathrm{Cu}$ & $0.83 \pm 0.98$ & $3.09 \pm 2.67$ & $7.47 \pm 2.14$ \\
$\mathrm{~Pb}$ & - & - & - \\
$\mathrm{Zn}$ & $0.05 \pm 0.04$ & $0.12 \pm 0.12$ & $6.51 \pm 2.26$ \\
Glifosato (principio activo)* & - & - & - \\
Glifosato ATANOR® & $0.11 \pm 0.06$ & $0.24 \pm 0.08$ & $12.08 \pm 3.15$ \\
$\mathrm{~S}$ acutus & & & \\
$\mathrm{Cu}$ & $0.17 \pm 0.13$ & $0.29 \pm 0.14$ & $10.92 \pm 3.76$ \\
$\mathrm{~Pb}$ & $0.04 \pm 0.04$ & $0.11 \pm 0.11$ & $>20$ \\
$\mathrm{Zn}$ & $0.05 \pm 0.05$ & $0.09 \pm 0.09$ & $>20$ \\
Glifosato (principio activo) & - & - & - \\
Glifosato ATANOR®* & - & - & - \\
\hline
\end{tabular}

*No se observaron diferencias significativas $(P<0.05)$ entre la menor concentración $(0.5 \mathrm{mg} / \mathrm{L})$ y el resto de las concentraciones ensayadas. 
Tabla 2. Valores de la pendiente de la curva sigmoidea (HS), del valor entre $0 \%$ y $100 \%$ de inhibición del crecimiento a, obtenidos para los respectivos ajustes de los datos de \% I al modelo sigmoidal, y sus respectivos coeficientes de determinación $\left(R^{2}\right)$ para cada especie algal y sustancia. Se muestran los estimadores HS y a \pm ES.

Table 2. Values of the sigmoid curve slope (HS), value between $0 \%$ and $100 \%$ of the inhibition growth a obtained for the respective adjustments of the \%I data to the sigmoidal model, and their respective coefficients of determination $\left(R^{2}\right)$ for each species and substance. HS estimators and $\mathrm{a} \pm \mathrm{SE}$ are displayed.

\begin{tabular}{lccc}
\hline Sustancia & $\mathrm{HS}$ & $\mathrm{a}$ & $R^{2}$ \\
\hline R. subcapitata & & & \\
$\mathrm{Cu}$ & $-1.40 \pm 1.40$ & $7.17 \pm 0.31$ & 0.85 \\
$\mathrm{~Pb}$ & $\mathrm{NA}$ & $\mathrm{NA}$ & $\mathrm{NA}$ \\
$\mathrm{Zn}$ & $0.79 \pm 1.04$ & $6.11 \pm 1.05$ & 0.65 \\
Glifosato (principio activo) & $\mathrm{NA}$ & $\mathrm{NA}$ & $\mathrm{NA}$ \\
Glifosato ATANOR® & $-0.94 \pm 0.14$ & $11.26 \pm 1.42$ & 0.75 \\
S. acutus & & & \\
$\mathrm{Cu}$ & $-1.9 \pm 1.55$ & $0.79 \pm 0.27$ & 0.75 \\
$\mathrm{~Pb}$ & $-0.38 \pm 0.15$ & $110 \pm 50.4$ & 0.3 \\
Zn & $-0.54 \pm 3.14$ & $11.26 \pm 3.41$ & 0.54 \\
Glifosato (principio activo) & $\mathrm{NA}$ & $\mathrm{NA}$ & $\mathrm{NA}$ \\
Glifosato ATANOR® & $\mathrm{NA}$ & $\mathrm{NA}$ & $\mathrm{NA}$ \\
\hline $\mathrm{NA}: \mathrm{n}$ ajusto a modelo & &
\end{tabular}

NA: no ajustó al modelo
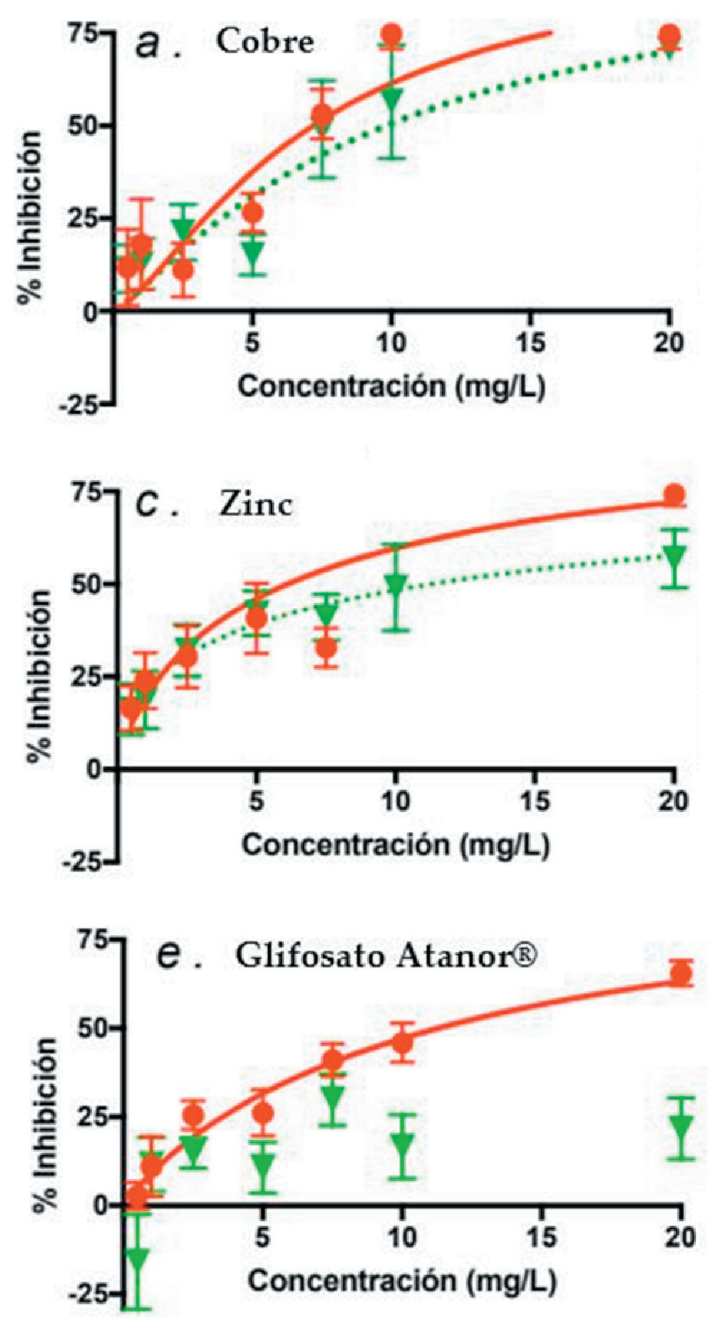
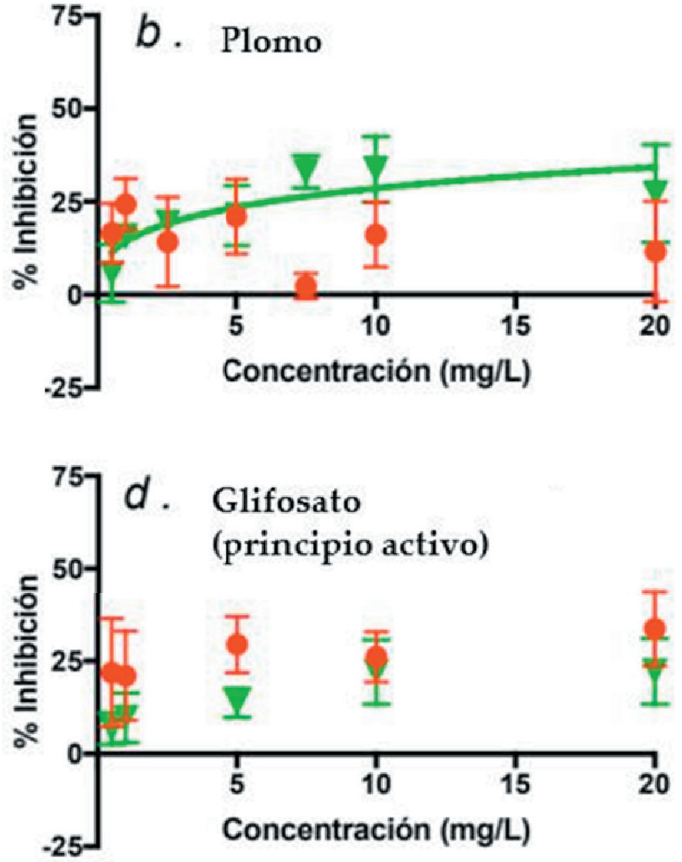

Figura 1. Curvas de inhibición del crecimiento poblacional de Raphidocelis subcapitata (línea entera y círculo en rojo) y Scenedesmus acutus (línea punteada y triángulo en verde) a concentraciones crecientes de las sustancias individuales a) cobre, b) plomo, c) zinc, d) glifosato (principio activo) y e) glifosato formulación ATANOR ${ }^{\circledR}$, con ajuste al modelo sigmoidal. Las barras de error representan el IC 95\%.

Figure 1. Population growth inhibition curves of Raphidocelis subcapitata (solid line and circle in red) and Scenedesmus acutus (dotted line and triangle in green) at increasing concentrations of the individual substances a) copper, b) lead, c) zinc, d) glyphosate (active ingredient) and e) glyphosate ATANOR® formulation, with fit to the sigmoidal model. The error bars represent the 95\% CI. 
del glifosato no afectó significativamente el crecimiento de las especies algales $(P>0.05)$.

Para evaluar los efectos de las mezclas binarias sobre el crecimiento de las algas se excluyó el principio activo del glifosato, ya que no resultó inhibidor en ninguna de las dos especies. En las tres mezclas binarias con $\mathrm{Cu}$, se observó estimulación del crecimiento a bajas concentraciones $(0.5$ y $1 \mathrm{mg} / \mathrm{L})$ en $R$. subcapitata (Figura 2). A bajas concentraciones de las mezclas se observó una mayor toxicidad en $S$. acutus que en $R$. subcapitata, a excepción de la mezcla $\mathrm{Zn}+\mathrm{Pb}$. Esta tendencia fue contrastante a concentraciones más altas, a excepción de

\section{a . Cobre + Zinc}

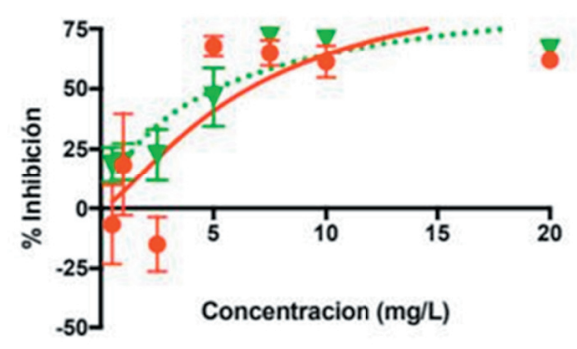

C. Cobre+glifosato Atanor ${ }^{\circledR}$

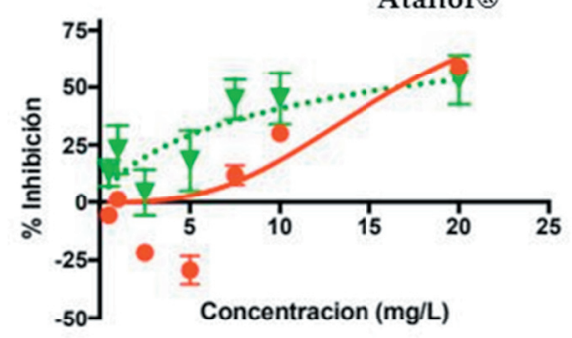

e. Pomo+glifosato Atanor ${ }^{\circledR}$

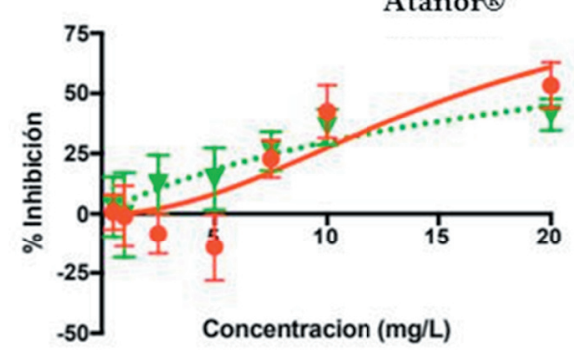

la mezcla $\mathrm{Cu}+\mathrm{Pb}$, en la que la toxicidad fue mayor que en la especie autóctona en todas las concentraciones, igualándose en la concentración más alta $(20 \mathrm{mg} / \mathrm{L})$ en ambas especies (Figura 2). En todas las mezclas se observaron valores más bajos de CE10 y CE20 en $S$. acutus que en $R$. subcapitata, mientras que las CE50 fueron similares en ambas especies (Tabla 3). S. acutus no presentó un efecto dosisrespuesta para la mezcla $\mathrm{Pb}+\mathrm{Zn}$ en el rango de concentraciones y condiciones de ensayo utilizados para $S$. acutus reflejándose en la falta de convergencia al ajuste al modelo de la curva sigmoidea. En las tres mezclas en las que uno de los componentes fue el glifosato ATANOR ${ }^{\circledR}$,

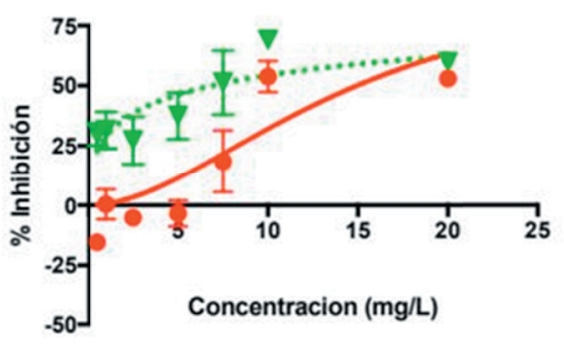

\section{d. Zinc+Plomo}

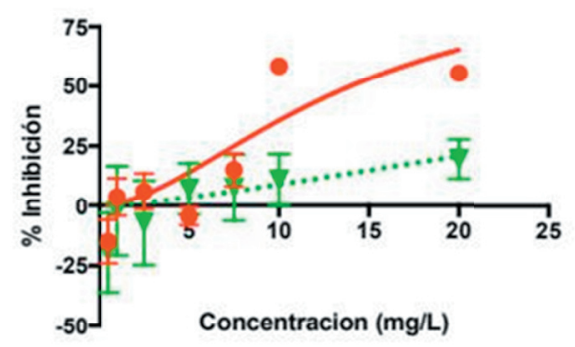

\section{f. Zinc+glifosato} Atanor ${ }^{\circledR}$

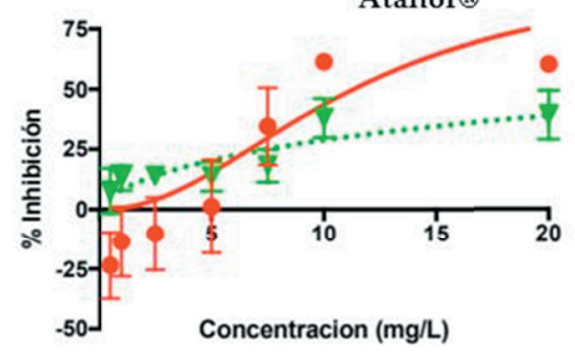

Figura 2. Curvas de inhibición del crecimiento poblacional de Raphidocelis subcapitata (línea entera y círculo en rojo) y Scenedesmus acutus (línea punteada y triángulo en verde) a concentraciones crecientes de las mezclas binarias. a) $\mathrm{Cu}+\mathrm{Zn}$; b) $\mathrm{Cu}+\mathrm{Pb}$; c) $\mathrm{Cu}+$ glifosato ATANOR $^{\oplus}$; d) $\mathrm{Zn}+\mathrm{Pb}$; e) Pb+glifosato ATANOR ${ }^{\circledR}$; f) Zn+glifosato ATANOR ${ }^{\circledR}$, con ajuste al modelo sigmoidal. Las barras de error representan el IC $95 \%$.

Figure 2. Population growth inhibition curves of Raphidocelis subcapitata (solid line and circle in red) and Scenedesmus acutus (dotted line and triangle in green) at increasing concentrations of binary mixtures: a) $\mathrm{Cu}+\mathrm{Zn} ; \mathrm{b}) \mathrm{Cu}+\mathrm{Pb} ; \mathrm{c}$ ) $\mathrm{Cu}+$ glyphosate ATANOR®; d) $\mathrm{Zn}+\mathrm{Pb}$; e) $\mathrm{Pb}+$ glyphosate ATANOR®; f) Zn+glyphosate ATANOR®, with adjustment to the sigmoidal model. The error bars represent the $95 \%$ CI. 
Tabla 3. Concentraciones efectivas 10, 20 y 50 (CE10, CE20 y CE50) de las mezclas binarias obtenidas para las especies algales R. subcapitata y S. acutus. Las concentraciones de las mezclas (1:1) se expresan en mg/L.

Table 3. Effective concentrations 10, 20 and 50 (EC10, EC20 and EC50) of the binary mixtures obtained for the algal species R. subcapitata and S. acutus. The concentrations of the mixtures (1:1) are expressed in $\mathrm{mg} / \mathrm{L}$.

\begin{tabular}{|c|c|c|c|}
\hline Mezcla & CE10 & CE20 & CE50 \\
\hline \multicolumn{4}{|l|}{ R. subcapitata } \\
\hline $\mathrm{Cu}+\mathrm{Pb}^{*}$ & $0.35 \pm 0.14$ & $0.55 \pm 0.11$ & $11.84 \pm 0.50$ \\
\hline $\mathrm{Cu}+\mathrm{Zn}^{*}$ & $0.17 \pm 0.14$ & $0.25 \pm 0.13$ & $8.23 \pm 4.10$ \\
\hline $\mathrm{Cu}+$ Glifosato ATANOR ${ }^{\circledast *}$ & $0.58 \pm 0.13$ & $0.79 \pm 0.07$ & $17.3 \pm 3.81$ \\
\hline $\mathrm{Pb}+\mathrm{Zn}$ & $0.32 \pm 0.16$ & $0.5 \pm 0.14$ & $15.99 \pm 5.47$ \\
\hline $\mathrm{Pb}+\mathrm{Glifosato}$ ATANOR ${ }^{\circledast}$ & $0.41 \pm 0.25$ & $0.55 \pm 0.18$ & $13.06 \pm 5.24$ \\
\hline Zn+Glifosato ATANOR ${ }^{\circledast}$ & $0.39 \pm 0.18$ & $0.59 \pm 0.13$ & $19.70 \pm 8.08$ \\
\hline \multicolumn{4}{|l|}{ S. acutus } \\
\hline $\mathrm{Cu}+\mathrm{Pb}$ & $0.02 \pm 0.02$ & $0.04 \pm 0.04$ & $11.25 \pm 7.57$ \\
\hline $\mathrm{Cu}+\mathrm{Zn}$ & $0.06 \pm 0.04$ & $0.12 \pm 0.06$ & $5.50 \pm 1.95$ \\
\hline $\mathrm{Cu}+\mathrm{Glifosato}$ ATANOR ${ }^{\circledast}$ & $0.12 \pm 0.12$ & $0.21 \pm 0.19$ & $>20$ \\
\hline $\mathrm{Pb}+\mathrm{Zn}$ & - & - & - \\
\hline $\mathrm{Pb}+\mathrm{Glifosato}$ ATANOR ${ }^{\circledast}$ & $0.13 \pm 0.12$ & $0.25 \pm 0.23$ & $>20$ \\
\hline Zn+Glifosato ATANOR ${ }^{\circledast}$ & $0.18 \pm 0.18$ & $0.31 \pm 0.25$ & $>20$ \\
\hline
\end{tabular}

*Se observó estimulación del crecimiento en las concentraciones 0.5 y $1 \mathrm{mg} / \mathrm{L}$.

Tabla 4. Valores de la pendiente de la curva sigmoidea (HS), del valor entre $0 \%$ y $100 \%$ de inhibición del crecimiento a, obtenidos para los respectivos ajustes de los datos de \% I al modelo sigmoidal, y coeficientes de determinación $\left(R^{2}\right)$ para cada especie algal y mezcla binaria. Se muestran los estimadores HS y a \pm ES.

Table 4. Values of the sigmoid curve slope (HS), value between $0 \%$ and $100 \%$ of the growth inhibition a obtained for the respective adjustments of the \% I data to the sigmoidal model, and coefficients of determination $\left(R^{2}\right)$ for each algal species and binary mixture. HS estimators and $\mathrm{a} \pm \mathrm{SE}$ are displayed.

\begin{tabular}{|c|c|c|c|}
\hline Mezcla binaria & HS & a & $R^{2}$ \\
\hline \multicolumn{4}{|l|}{ R. subcapitata } \\
\hline $\mathrm{Cu}+\mathrm{Pb}$ & $-1.87 \pm 0.48$ & $14.94 \pm 2.11$ & 0.65 \\
\hline $\mathrm{Cu}+\mathrm{Zn}$ & $-1.38 \pm 0.50$ & $6.55 \pm 1.46$ & 0.52 \\
\hline $\mathrm{Cu}+$ Glifosato ATANOR ${ }^{\circledast}$ & $-2.93 \pm 0.77$ & $16.62 \pm 1.63$ & 0.66 \\
\hline $\mathrm{Pb}+\mathrm{Zn}$ & $-1.77 \pm 0.49$ & $13.46 \pm 2.02$ & 0.69 \\
\hline $\mathrm{Pb}+\mathrm{Glifosato}$ ATANOR ${ }^{\circledR}$ & $-2.05 \pm 0.68$ & $16.08 \pm 2.76$ & 0.48 \\
\hline Zn+Glifosato ATANOR ${ }^{\circledR}$ & $-2.09 \pm 0.84$ & $11.32 \pm 2.11$ & 0.47 \\
\hline \multicolumn{4}{|l|}{ S. acutus } \\
\hline $\mathrm{Cu}+\mathrm{Pb}$ & $-0.46 \pm 0.13$ & $6.87 \pm 2.23$ & 0.37 \\
\hline $\mathrm{Cu}+\mathrm{Zn}$ & $-0.87 \pm 0.17$ & $5.12 \pm 0.88$ & 0.68 \\
\hline $\mathrm{Cu}+\mathrm{Glifosato}$ ATANOR ${ }^{\circledR}$ & $-0.73 \pm 0.24$ & $16.57 \pm 6.13$ & 0.35 \\
\hline $\mathrm{Pb}+\mathrm{Zn}$ & NA & NA & NA \\
\hline $\mathrm{Pb}+\mathrm{Glifosato}$ ATANOR ${ }^{\circledast}$ & $-0.93 \pm 0.38$ & $25.03 \pm 11.82$ & 0.33 \\
\hline Zn+Glifosato ATANOR ${ }^{\circledast}$ & $-0.65 \pm 0.21$ & $39.65 \pm 22.49$ & 0.35 \\
\hline
\end{tabular}

NA: no ajustó al modelo

las CE50 fueron mayores a $20 \mathrm{mg} / \mathrm{L}$ para $S$. acutus. En estos casos no pudo obtenerse un valor de concentración, ya que no se produjo inhibición del crecimiento de las algas en el rango de concentración utilizada.

Desde una perspectiva general, la cepa autóctona de $S$. acutus presentó ajustes notablemente más débiles que la cepa estándar de $R$. subcapitata (Tabla 4). Ningún ajuste explicó más del $40 \%$ de la variación del
\%I con la excepción de la mezcla $\mathrm{Cu}+\mathrm{Zn}$, que presentó el mejor ajuste de esa serie $\left(R^{2}=0.68\right)$. Los valores de las pendientes estimadas para S. acutus fueron más bajas (Tabla 4).

Para evaluar los posibles efectos sinérgicos o antagónicos de las mezclas sobre las algas se calcularon las unidades tóxicas UT20 y UT50 a partir de las respectivas CE20 y CE50 de las mezclas binarias dividido las CE20 y CE50 de los tóxicos individuales (Tabla 5). Los valores 
Tabla 5. Unidades Tóxicas (UT) obtenidas para las mezclas binarias a partir de las CE20 y CE50 de las sustancias individuales y de las mezclas binarias para las especies R. subcapitata y S. acutus.

Table 5. Toxic Units (TU) obtained for the binary mixtures from the EC20 and EC50 of the individual substances and binary mixtures for the algal species R. subcapitata and S. acutus.

\begin{tabular}{|c|c|c|c|c|}
\hline Mezcla & CE20 & CE50 & UT20 & UT50 \\
\hline \multicolumn{5}{|l|}{ R. subcapitata } \\
\hline $\mathrm{Cu}$ & 3.09 & 7.47 & & \\
\hline $\mathrm{Zn}$ & 2.67 & 6.51 & & \\
\hline Glifosato ATANOR $^{\circledast}$ & 0.24 & 12.08 & & \\
\hline $\mathrm{Cu}+\mathrm{Zn}$ & 0.25 & 8.23 & 2.16 & 2.35 \\
\hline $\mathrm{Cu}+$ Glifosato ATANOR ${ }^{\circledR}$ & 0.79 & 17.3 & 3.54 & 3.75 \\
\hline Zn+Glifosato ATANOR ${ }^{\circledR}$ & 0.59 & 19.70 & 6.87 & 3.08 \\
\hline \multicolumn{5}{|l|}{ S. acutus } \\
\hline $\mathrm{Cu}$ & 0.29 & 10.92 & & \\
\hline $\mathrm{Pb}$ & 0.11 & - & & \\
\hline $\mathrm{Zn}$ & 0.09 & - & & \\
\hline $\mathrm{Cu}+\mathrm{Pb}$ & 0.04 & - & 0.47 & - \\
\hline $\mathrm{Cu}+\mathrm{Zn}$ & 0.12 & - & 1.74 & - \\
\hline
\end{tabular}

de UT20 y UT50 determinados en el caso de $R$. subcapitata mostraron efectos antagónicos en las tres mezclas obteniéndose valores de CE20 y CE50 tanto en las mezclas como en las sustancias individuales, es decir $\mathrm{Cu}+\mathrm{Zn}$, $\mathrm{Cu}+$ glifosato ATANOR ${ }^{\circledR}$ y Zn+glifosato ATANOR $^{\circledR}$. Los valores de UT fueron más altos cuando la formulación del glifosato estuvo presente en la mezcla. En el caso de S. acutus no pudieron calcularse las UT50 debido a la ausencia de valores de CE50 para las sustancias individuales (con la excepción de $\mathrm{Cu}$ ), y por lo tanto sólo se calcularon las UT20. En este caso el glifosato ATANOR ${ }^{\circledR}$ no mostró un efecto dosis-respuesta sobre el crecimiento de las algas al enfrentarlo de manera individual. Solo dos mezclas permitieron el abordaje desde las $\mathrm{UT}$, la mezcla $\mathrm{Cu}+\mathrm{Pb}$, que presentó un valor de $\mathrm{UT}<1$ y la mezcla $\mathrm{Cu}+\mathrm{Zn}$, con un valor de $\mathrm{UT}>1$ (Tabla 5). Esto indica que la primera produjo un efecto sinérgico sobre la especie autóctona y la segunda un efecto antagónico.

\section{DISCUSIÓN}

Las CE50 obtenidas para los metales $\mathrm{Cu}$ y Zn en $R$. subcapitata $(7.47$ y $6.51 \mathrm{mg} / \mathrm{L}$, respectivamente) fueron considerablemente más altas que las reportadas en la literatura (entre 0.01 y $0.13 \mathrm{mg} / \mathrm{L}$ para el $\mathrm{Cu}$ y entre 0.05 y $0.31 \mathrm{mg} / \mathrm{L}$ para el $\mathrm{Zn}$ ) para dicha especie (Guéguenetal.2004; Geisetal.2000; Magdaleno et al. 2014). Por otra parte, dichos autores reportaron valores de CE50 para el $\mathrm{Pb}$ de entre 0.29 y $2.7 \mathrm{mg} / \mathrm{L}$, mientras que en el presente trabajo no se obtuvo un efecto dosis-respuesta a lo largo del rango de concentraciones 0.5 a $20 \mathrm{mg} / \mathrm{L}$. Una de las ventajas de la utilización de los bioensayos con algas para evaluar la calidad de aguas superficiales es que los mismos proporcionan información tanto de la inhibición del crecimiento producida por la presencia de tóxicos como de su estimulación debida a la concentración de nutrientes (Magdaleno et al. 2018). En este trabajo, los bioensayos se llevaron a cabo utilizando un medio de cultivo rico en nutrientes (BBM), que contiene concentraciones mayores de $\mathrm{N}$ y $\mathrm{P}$ que los medios utilizados en otros trabajos. La estimulación del crecimiento debido a las concentraciones elevadas de nutrientes contrarrestaría parcialmente el efecto inhibitorio de los metales en solución, reflejándose en los valores altos de CE50 obtenidos. Cabe destacar que la utilización de un medio de cultivo rico en nutrientes, resulta más satisfactorio para establecer condiciones similares a las de los ríos y arroyos de la Provincia de Buenos Aires, que generalmente son eutróficos. Por ejemplo, en el arroyo Burgos (localidad de San Pedro), de donde fue aislada la cepa de la especie $S$. acutus, se encontraron concentraciones altas de N y P (14.2 y 14.9 mg/L, respectivamente) (Bollani et al. 2019).

Por otra parte, un componente que se incluye en el medio de cultivo como quelante de los iones micronutrientes, el etilendiaminotetraacético (EDTA), suele formar complejos con los metales tóxicos, lo que disminuye su biodisponibilidad y, por lo tanto, su toxicidad. Esto explicaría los valores elevados de CE50 del Cu y Zn. Asimismo, las aguas naturales poseen sustancias orgánicas en solución (materia orgánica disuelta), compuesta por ácidos húmicos y fúlvicos, los 
cuales forman complejos con iones metálicos. Se ha discutido acerca de la inclusión de EDTA en los medios de cultivo utilizados para bioensayos con algas cuando se desea evaluar la toxicidad de metales pesados (Geis et al. 2000). Sin embargo, el protocolo de la agencia de Canadá sugiere incluirlo (Environmental Canada 2007). Así también, Domingos et al. (2014) sugieren la necesidad de incluir los complejos naturales orgánicos e inorgánicos en el análisis de la toxicidad de los metales pesados para garantizar una protección ambiental adecuada. De este modo, la inclusión del EDTA en el medio de cultivo acercaría más los resultados a las condiciones ambientales.

Los bioensayos con cepas algales autóctonas, aisladas de los ambientes naturales de interés, proporcionan resultados más realistas acerca de la toxicidad de las sustancias presentes en dichos ambientes. Sin embargo, es conveniente comparar esos resultados con los obtenidos para una especie estándar en las mismas condiciones de ensayo (Carusso et al. 2018). En este trabajo, las CE50 obtenidas para los metales $\mathrm{Cu}$ y $\mathrm{Zn}$ fueron mayores en la especie autóctona que en la especie estándar (Tabla 1), lo cual indica una mayor resistencia de S. acutus a dichos tóxicos. Sin embargo, las CE10 y CE20 de esas sustancias (Tabla 1) y de todas las mezclas binarias analizadas (Tabla 3), fueron más bajas en $S$. acutus que en $R$. subcapitata, lo cual indicaría una mayor sensibilidad de la especie autóctona a bajas concentraciones de los tóxicos y sus mezclas. Según Beasley et al. (2015), las CE10 pueden compararse a las concentraciones de no efecto (NOEC), es decir las concentraciones por encima de las cuales se produciría un efecto tóxico observable. De este modo, las CE10 podrían considerarse ambientalmente relevantes, ya que establecen un umbral de protección para los organismos.

Los metales pesados pueden afectar el crecimiento de las algas de diversas formas al inhibir diferentes procesos fisiológicos. La fotosíntesis en particular, es un proceso sensible al estrés ambiental. El $\mathrm{Cu}$ y el $\mathrm{Zn}$ son metales esenciales para las algas, ya que participan en varios procesos fisiológicos tales como la fotosíntesis y la respiración (Starodub and Wong 1987). Sin embargo, cuando estos elementos se encuentran en concentraciones altas pueden resultar tóxicos e inhibir el crecimiento poblacional (Franklin et al. 2002). El Pb no desempeña ninguna función celular, sin embargo puede ser incorporado a las proteínas celulares mediante uniones a grupos sulfhídrico (SH) y puede desplazar a otros iones metálicos como el magnesio $(\mathrm{Mg})$, el calcio (Ca) y el hierro (Fe), los cuales están involucrados en el aparato fotosintético y en la fotosíntesis (Lamelas et al. 2009).

Las concentraciones máximas de $\mathrm{Cu}, \mathrm{Pb}$ y Zn encontradas en las aguas superficiales del Arroyo Burgos, de donde fue aislada la cepa de la especie $S$. acutus, fueron $0.252 \mathrm{mg} / \mathrm{L}$, $0.176 \mathrm{mg} / \mathrm{L}$ y $0.960 \mathrm{mg} / \mathrm{L}$, respectivamente (Bollani et al. 2019). Estas concentraciones son mayores que las CE10 y CE20 obtenidas para S. acutus, pero menores que las CE20 obtenidas para $R$. subcapitata. La mayor respuesta inhibitoria observada en $S$. acutus a las concentraciones ambientales de metales pesados resulta indicadora de su potencialidad para la evaluación de la contaminación de las aguas superficiales de zonas rurales mediante bioensayos a corto plazo. S. acutus podría presentar mecanismos de adaptación para tolerar niveles elevados de metales pesados, como por ejemplo por detoxificación o exclusión de estos tóxicos, tal como lo describen algunos autores para diferentes especies de algas verdes (Janssen and Heijerick 2003; Kalinowska and Pawlik-Skowronska 2010; Piotrowska-Niczyporuk et al. 2012). En primer lugar, la pared celular juega un rol importante de defensa impidiendo el ingreso de metales pesados a la célula, ya sea mediante la adsorción de iones metálicos en la superficie externa o a través de la liberación de ligandos extracelulares (exopolisacáridos) (Sabatini et al. 2009; Kalinowska and Pawlik-Skowronska 2010). Además, la presencia tanto de péptidos intracelulares ricos en azufre (fitoquelatinas) como de glutatión favorece el desarrollo de mecanismos de desintoxicación y reducción del daño por estrés oxidativo provocado por los iones metálicos sean esenciales o no (Sabatini et al. 2009; Piotrowska-Niczyporuk et al. 2012).

El principio activo del glifosato no resultó tóxico para las dos cepas algales en las condiciones y concentraciones ensayadas, mientras que $R$. subcapitata experimentó una inhibición significativa del crecimiento al enfrentarse con el glifosato ATANOR ${ }^{\circledR}$ en concentraciones mayores a $10 \mathrm{mg} / \mathrm{L}$ (Figura 1). Algunas evidencias indicarían que los aditivos que se agregan a las formulaciones de herbicidas (e.g., surfactantes, metales pesados, entre otros) pueden resultar más tóxicos para las algas que el principio activo del herbicida en cuestión (González et al. 2019). 
La toxicidad de los metales $\mathrm{Cu}, \mathrm{Pb}$ y $\mathrm{Zn}$, y del glifosato ATANOR ${ }^{\circledR}$ fue mayor de manera individual que en las mezclas binarias, según surge de los resultados de CE50, aunque en el caso de $S$. acutus la mezcla $\mathrm{Cu}+\mathrm{Zn}$ tuvo mayor efecto que los metales ensayados de manera individual (Tablas 1 y 3). El análisis de las UT para evaluar la interacción entre las sustancias ensayadas evidenció que en $R$. subcapitata todas las mezclas tuvieron valores de UT $>1$, indicando una relación antagónica entre los componentes de cada mezcla, incluso en combinación con el glifosato ATANOR ${ }^{\circledR}$, evidenciando una relación antagónica entre el herbicida y los metales Cu y Zn (Tabla 5). La mezcla de $\mathrm{Cu}$ y $\mathrm{Zn}$ para esta cepa también resultó mayor a 1 (UT=2.34). Según Starodub y Wong (1987), en algas verdes las mezclas binarias $\mathrm{Cu}+\mathrm{Zn}$ actúan de manera antagónica a concentraciones entre 0.1 y $0.5 \mathrm{mg} / \mathrm{L}$. En el caso de $S$. acutus, se observó una relación sinérgica a bajas concentraciones de la mezcla $\mathrm{Cu}+\mathrm{Pb}$, semejantes a los niveles hallados en las aguas superficiales del Arroyo Burgos (Bollani et al. 2019).

Las algas son componentes esenciales de los ecosistemas acuáticos, principales productores primarios planctónicos y alimento para otros organismosdelplancton, peceseinvertebrados. El impacto de los contaminantes sobre las algas afectaría directamente la estructura y función del ecosistema. En el presente trabajo se evaluaron los efectos de algunos contaminantes sobre poblaciones de algas cultivadas en el laboratorio. Es importante tener en cuenta que la extrapolación de los resultados de laboratorio a la dinámica natural tiene sus limitaciones, ya que la complejidad de los procesos de las aguas naturales no puede emularse en las condiciones estándar utilizadas en los ensayos. Por ejemplo, los medios de cultivo no contienen materia orgánica disuelta, contienen grandes cantidades de nutrientes esenciales y tienen un $\mathrm{pH}$ relativamente alto. Por otra parte, los bioensayos de laboratorio se realizan con una sola especie, lo que dificulta establecer un efecto a nivel de las comunidades naturales, por ejemplo el fitoplancton natural. Sin embargo, a pesar de las limitaciones mencionadas, la realización de bioensayos utilizando cepas algales autóctonas, adaptadas a condiciones ambientales particulares, resulta más apropiado para inferir posibles respuestas en el sistema natural. Dada su sensibilidad a concentraciones ambientales de metales pesados, la cepa de la especie $S$. acutus podría ser incorporada como un nuevo organismo de ensayo para el análisis de la calidad de aguas en zonas naturales. Además, según surge de este trabajo, es necesario analizar los efectos de las interacciones entre las distintas sustancias detectadas en los cuerpos de agua a fin de establecer límites de calidad más adecuados para la zona de estudio.

Agradecimientos. Este estudio fue realizado a través de los subsidios UBACyT $\mathrm{N}^{\circ}$ 20020150200116BA y UBACyT $\mathrm{N}^{\circ}$ 20020130100601BA otorgados por la Universidad de Buenos Aires, Argentina.

\section{REFERENCIAS}

Alloway, B. J. 1995. Heavy metals in soils. Blackie Academic and Professional, London. Pp. 235-274. https://doi.org/ 10.1007/978-94-011-1344-1.

Aparicio, V. C., E. De Gerónimo, D. Marino, J. Primost, P. Carriquiriborde, and J. L. Costa. 2013. Environmental fate of glyphosate and aminomethylphosphonic acid in surface waters and soil of agricultural basins. Chemosphere 93(9): 1866-1873. https://doi.org/10.1016/j.chemosphere.2013.06.041.

Archibald, P. A., and H. C. Bold. 1970. Phycological Studies. XI. The Genus Chlorococcum Meneghini. Univ. Texas Public., N7015, Austin, Texas. Pp. 86.

Beasley, A., S. E. Belanger, J. L. Brill, and R. R. Otter. 2015. Evaluation and comparison of the relationship between NOEC and EC10 or EC20 values in chronic Daphnia toxicity testing. Environ Toxicol Chem 34:2378-2384. https: //doi.org/10.1002/etc.3086.

Blinova, I. 2004. Use of freshwater algae and duckweeds for phytotoxicity testing. Environ Toxicol 19(4):425-428. https: //doi.org/10.1002/tox.20042.

Bollani, S., L. de Cabo, C. Chagas, J. Moretton, C. Weigandt, A. Fabrizio de Iorio, and A. Magdaleno. 2019. Genotoxicity of water samples from an area of the Pampean region (Argentina) impacted by agricultural and livestock activities. Environ Sci Pollut Res 26(27):27631-27639. https://doi.org/10.1007/s11356-018-3263-9.

Broderius, S. J., M. C. Kahl, G. E. Elonen, D. E. Hammermeister, and M. D. Hoglund. 2005. A comparison of the lethal and sublethal toxicity of organic chemical mixtures to the feathed minnow (Pimephales promelas). Environ Toxicol Chem 24(12):3117-27. https://doi.org/10.1897/05-094R.1.

Carusso, S., A. B. Juárez, J. Moretton, and A. Magdaleno. 2018. Effects of three veterinary antibiotics and their binary mixtures on two green alga species. Chemosphere 194:821-827. https://doi.org/10.1016/j.chemosphere.2017.12.047.

Domingos, R. F., A. Gelabert, S. Carreira, A. Cordeiro, Y. Sivry, and M. F. Benedetti. 2014. Metals in the Aquatic Environment-Interactions and Implications for the Speciation and Bioavailability: A Critical Overview. Aquat 
Geochem 21(2-4):1-27. https://doi.org/10.1007/s10498-014-9251-x.

Duke, S. O. 1988. Herbicides: chemistry, degradation and mode of action. Pp. 1-70 in P. C. Kearney and D. D. Kaufman (eds.). Marcel Dekker, USA.

Environmental Canada. 2007. Biological test method: growth inhibition test using a freshwater algae. EPS 1/RM/25, Second Ed. Pp. 53.

Fergusson, J. 1990. The heavy elements. Chemistry, environmental impact and health effects. Pergamon Press, Oxford. Pp 175-182.

Franklin, N. M., J. L. Stauber, R. P. Lim, P. Petocz. 2002. Toxicity of metal mixtures to a tropical freshwater alga (Chlorella $\mathrm{sp}$.): the effect of interactions between copper, cadmium, and zinc on metal cell binding and uptake. Environ Toxicol Chem 21(11):2412-2422. https://doi.org/10.1897/1551-5028(2002)021\%3C2412:TOMMTA\%3E2.0.CO;2. https://doi.org/ 10.1002/etc.5620211121.

Geis, W. S., K. L. Fleming, E. T. Korthals, G. Searle, L. Reynolds, and D. A. Karner. 2000. Modifications to the algal growth inhibition test for use as a regulatory assay. Environ Toxicol Chem 19(1):36-40. https://doi.org/10.1002/ etc.5620190105.

Guéguen, C., R. Gilbin, M. Pardos, and J. Dominik. 2004. Water toxicity and metal contamination assessment of a polluted river: the Upper Vistula River (Poland). Appl Geochem 19:153-162. https://doi.org/10.1016/S0883-2927(03)00110-0.

González, D., A. B. Juárez, C. P. Krugn, M. Santos, and S. Vera. 2019. Freshwater periphyton response to technical-grade and two commercial formulations of glyphosate. Ecol Austral 29:20-27. https://doi.org/10.25260/EA.19.29.1.0.816.

González-Pleiter, M., S. Gonzalo, I. Rodea-Palomares, F. Leganés, R. Rosal, K. Boltes, E. Marco, and F. FernándezPiñas. 2013. Toxicity of five antibiotics and their mixtures towards photosynthetic aquatic organisms: implications for environmental risk assessment. Wat Res 47:2050-2064. https://doi.org/10.1016/j.watres.2013.01.020.

ISO. 2009. Water quality - Freshwater Algal Growth Inhibition Test with Unicellular Green Algae, revision. International Standardization Organization, Brussels (ISO 8692).

Janssen, C. R., and D. G. Heijerick. 2003. Algal toxicity tests for environ- mental risk assessments of metals. Rev Environ Contam Toxicol 178:23-52. https://doi.org/10.1007/0-387-21728-2_2.

Jorgensen, S. E., B. H. Sørensen, and H. Mahler. 1997. Handbook of estimation methods in ecotoxicology and environmental chemistry (Vol. 2). CRC Press.

Kahru, A., A. Ivask, K. Kasemets, L. Pollumaa, I. Kurvet, M. François, and H. C. Dubourguier 2005. Biotest and biosensors in ecotoxicological risk assessment of field soils polluted with zinc, lead, and cadmium. Environ Toxicol Chem 24(11): 2973-2982. https://doi.org/10.1897/05-002R1.1.

Kalinowska, R., and B. Pawlik-Skowronska. 2010. Response of two terrestrial green microalgae (Chlorophyta, Trebouxiophyceae) isolated from Cu-rich and unpolluted soils to copper stress. Environ Pollut 158:2778-2785. https: //doi.org/10.1016/j.envpol.2010.03.003.

Kim, R. Y., J. K. Yoon, T. S. Kim, J. E. Yang, G. Owens, and K. R. Kim. 2015. Bioavailability of heavy metals in soils: definitions and practical implementation - a critical review. Environ Geochem Health 37:1041-1061. https://doi.org/ 10.1007/s10653-015-9695-y.

Küpper, H., I. Setlik, M. Spiller, F. S. Küpper, and O. Prášil. 2002. Heavy metal-induced inhibition of photosynthesis: targets of in vivo heavy metal chlorophyll formation. J Phycol 38(3):429-441. https://doi.org/10.1046/j.1529-8817.2002.t011-01148.x. https://doi.org/10.1046/j.1529-8817.2002.01148.x.

Lamelas, C., J. P. Pinheiro, and V. I. Slaveykova. 2009. Effect of humic acid on Cd (II), Cu (II), and Pb (II) uptake by freshwater algae: kinetic and cell wall speciation considerations. Environ Sci Technol 43(3):730-735. https://doi.org/ 10.1021/es802557r.

Magdaleno, A., L. de Cabo, S. Arreghini, and C. Salinas. 2014. Assessment of heavy metal contamination and water quality in an urban river from Argentina. Braz J Aquat Sci Tech 18(1):113-120. https://doi.org/10.14210/bjast.v18n1.p113120.

Magdaleno, A., M. Paz, J. Mantovano, L. de Cabo, S. Bollani, C. Chagas, L. Núñez, C. Tornello, and J. Moretton. 2018. Evaluación del impacto de las actividades rurales en la calidad del agua de la microcuenca del arroyo Burgos (San Pedro, Provincia de Buenos Aires). Rev Mus Argent Cienc Nat 20(2):239-250. https://doi.org/10.22179/REVMACN.20.588.

Nowell, L. H., J. E. Norman, P. W. Moran, J. D. Martin, and W. W. Stone. 2014. Pesticide Toxicity Index - A tool for assessing potential toxicity of pesticide mixtures to freshwater aquatic organisms. Sci Total Environ 476-477:144-157. https://doi.org/10.1016/j.scitotenv.2013.12.088.

Omar, H. H. 2002. Bioremoval of zinc ions by Scenedesmus obliquus and Scenedesmus quadricauda and its effects on growth and metabolism. Int Biodeter Biodegr 50:95-100. https://doi.org/10.1016/S0964-8305(02)00048-3.

Pérez, G. L., A. Torremorell, P. Mugni, P. Rodríguez, M. S. Vera, M. Do Nascimento, L. Allende, J. Bustingorry, R. Escaray, M. Ferraro, I. Izaguirre, H. Pizarro, C. Bonetto, D. P. Morris, and H. Zagarese. 2007. Effects of the herbicide Roundup on freshwater microbial communities: a mesocosm study. Ecol Appl 17:2310-2322. https://doi.org/10.1890/ 07-0499.1.

Peruzzo, P. J., A. A. Porta, and A. E. Ronco. 2008. Levels of glyphosate in surface waters, sediments and soils associated with direct sowing soybean cultivation in north pampasic region of Argentina. Environ Pollut 156:61-66. https: //doi.org/10.1016/j.envpol.2008.01.015.

Pignata, M. L., G. L. Gudiño, E. D. Wannaza, R. R. Plá, C. M. González, H. A. Carreras, and L. Orellana. 2002. Atmospheric quality and distribution of heavy metals in Argentina employing Tillandsia capillaris as a biomonitor. Environ Pollut 120:59-68. https://doi.org/10.1016/S0269-7491(02)00128-8. 
Piotrowska-Niczyporuk, A., A. Bajguz, E. Zambrzycka, and B. Godlewska- Zylkiewicz. 2012. Phytohormones as regulators of heavy metal biosorption and toxicity in green alga Chlorella vulgaris (Chlorophyceae). Plant Physiol Biochem 52:52-65. https://doi.org/10.1016/j.plaphy.2011.11.009.

Pizarro, H., M. S. Vera, A. Vinocur, G. Pérez, M. Ferraro, R. M. Helman, and M. dos Santos Afonso. 2015. Glyphosate input modifies microbial community structure in clear and turbid freshwater systems. Environ Sci Pollut Res 23(6): 5143-5153. https://doi.org/10.1007/s11356-015-5748-0.

Romero, D., M. C. Ríos de Molina, and A. B. Juárez. 2011. Oxidative stress induced by a commercial glyphosate formulation in a tolerant strain of Chlorella kessleri. Ecotoxicol Environ Saf 74:741-747. https://doi.org/10.1016/ j.ecoenv.2010.10.034.

Sabatini, S. E., A. B. Juárez, M. R. Eppis, L. Bianchi, C. M. Luquet, and M. C. Ríos de Molina. 2009. Oxidative stress and antioxidant defenses in two green microalgae exposed to copper. Ecotox Environ Safe 72:1200-1206. https: //doi.org/10.1016/j.ecoenv.2009.01.003.

Sarandón, S. J. 2013. Uso de agroquímicos en la Provincia de Buenos Aires. Relevamiento de la utilización de Agroquímicos en la Provincia de Buenos Aires - Mapa de Situación e incidencias sobre la salud. Informe de la Defensoría del Pueblo de la Provincia de Buenos Aires. Fac Cs Agrarias, UNLP. Pp. 246.

Starodub, M. E. and P. T. S. Wong. 1987. Short-term and long-term studies on individual and combined toxicities of copper, zinc and lead to Scenedesmus quadricauda. The Sci Total Environ 63:101-110. https://doi.org/10.1016/00489697(87)90039-8.

Suzuki, S., H. Yamaguchi, N. Nakajima, and M. Kawachi. 2018. Raphidocelis subcapitata (=Pseudokirchneriella subcapitata) provides an insight into genome evolution and environmental adaptations in the Sphaeropleales. Scientific Reports 8(1):1-13. https://doi.org/10.1038/s41598-018-26331-6.

USEPA. 2002. Selenastrum capricornutum growth test. In Short-term method for estimating the chronic toxicity of effluents and receiving water to freshwater organisms. USA.

Vera, M. S., L. Lagomarsino, M. Sylvester, G. L. Pérez, P. Rodríguez, H. Mugni, R. Sinistro, M. Ferraro, C. Bonetto, H. Zagarese, and H. Pizarro. 2010. New evidences of Roundup Max® (glyphosate formulation) impact on the periphyton community and the water quality of freshwater ecosystems. Ecotoxicology 19:713-719. https://doi.org/10.1007/s10646009-0446-7. 